Editorial

\title{
Bioengineering Materials for Environment Protection in a Changing Climate
}

\author{
Donatella Termini $\mathbb{D}^{1},{ }^{1}$ Hazi M. Azamathulla, ${ }^{2}$ and Jose R. B. Cantalice $\mathbb{i}^{3}$ \\ ${ }^{1}$ Department of Engineering, University of Palermo, Palermo, Italy \\ ${ }^{2}$ Department of Civil and Environmental Engineering, University of the West Indies, West Indies, Mona, Jamaica \\ ${ }^{3}$ Environmental Engineering Graduate Program, Rural Federal, University of Pernambuco, Recife, Brazil
}

Correspondence should be addressed to Donatella Termini; donatella.termini@unipa.it

Received 15 April 2019; Accepted 15 April 2019; Published 2 May 2019

Copyright (C) 2019 Donatella Termini et al. This is an open access article distributed under the Creative Commons Attribution License, which permits unrestricted use, distribution, and reproduction in any medium, provided the original work is properly cited.

Climate change is one of the biggest problems of our times, and as time passes, climate extremes tend to increase both in magnitude and in frequency. Because the severity of the extreme climate events has increased, the interaction of these events with vulnerable human and natural systems has more frequently lead to disasters. Recent climate models' projections have also shown a further substantial warming in temperature extremes by the end of the 21 st century. The prevision models' results also indicate that the global warming and the increase in frequency of temperature extremes could be strictly related to the anthropogenic forcing.

One of the environmental problems determined by warmer temperatures is the altering of the hydrological regime which determines changes in precipitation levels and patterns. The severity of disasters of hydrogeological origin produces serious damages to physical elements, buildings, and infrastructures located in vulnerable locations. Thus, climate-related disasters have both social and environmental dimensions.

The point is that although risks from climate extremes to industries, environment, and communities are increasing, still today they are not well quantified.

In this context, the knowledge of the potential environmental and socioeconomic impacts of climate change is fundamental to define procedures and technical solutions to protect the environment or to control the environmental hazards. In the ambit of the environmental engineering, which is the branch of engineering focusing on the aforementioned procedures and technical solutions, advanced bioengineering materials and combined techniques are needed to reduce the human impact on the environment. The present special issue collects contributions representing new ideas on innovative materials and advanced technologies in the area of environmental engineering.

A brief review of two techniques of controlling the human impact both in the extraurban and in urban areas, which adopt vegetation as living material, is reported in the paper by Termini. In particular, the first technique consists in using vegetation for soil erosion protection and rivers' bank stabilization in extraurban areas; the second technique consists in using vegetation for green roofs or walls added to buildings in urban areas. The discussion regarding the advantages and the disadvantages in adopting these two techniques concludes the review. The work demonstrates that the use of vegetation as biomaterial in environmental engineering could exert an important role in mitigating the worst effects due to anthropogenic actions. Furthermore, its use offers significant economic benefits, including a longer measure's life and an increasing biodiversity.

Another important issue for controlling soil degradation, deterioration, and improving the arid and semiarid soils is related to the use of polymers and biopolymers as soil-stabilizing additives. In the last two decades, the biodegradable polymers have been widely investigated to replace synthetic polymers. Dintcheva et al. analyze the effect of naturally occurring compounds, such as quercetin (Q) and sodium montmorillonite (NaMMt) at different weight ratios, in biopolyesters, such as polylactic acid, as suitable candidates for soil stabilization in the agricultural sector. The study is conducted through experimental and theoretical analyses by investigating the rheological behavior of these 
materials and applying the fractional-order calculus to fit the experimental data. As a result, the authors conclude that the fractional model is able to predict the rheological behavior of the investigated complex biopolymer systems especially at long experimental time, thus confirming that it is possible to consider them as suitable materials for soil stabilization.

Oueslati and Meftah focus on the use of substances, like the montmorillonite, as a green solution in the context of geological barrier for industrial and/or radioactive hazardous waste confinement. In particular, the work of Oueslati and Meftah investigates the water-montmorillonite interaction under variable atmospheric conditions, in order to characterize the interlamellar space configuration for its possible use. Atmospheric stress and hydrological disturbance are obtained at the laboratory scale. The study also indicates the limits observed during sorption/desorption processes in the case of the stressed sample.

Another problem related to the environment responses due to climate and anthropogenic impacts is related to the absorption properties of inorganic or organic suspended particulate materials in a water body. In fact, a variability in absorption coefficients has been documented in oceanic waters where phytoplankton and their derivative products (such as organic detritus, natural decay of the algal cells) are optically dominant. Wang et al. explore the spectral variation of suspended particulate material absorption and its changing mechanism during flood periods of Poyang Lake and the relative influence in the remote sensing monitoring. The authors separate absorption coefficients of suspended particulate materials in Poyang Lake through controlled laboratory experiments during a flood period and analyze the changing mechanism for corresponding components of absorption in order to facilitate improvements in the parameterization of remote sensing algorithms for the interpretation of remote sensing information. Results show that the nonlinear dependency of specific phytoplankton particulate absorption on pigment concentration for various trophic statuses in different periods could bring uncertainties to the application of remote sensing algorithms.

In industrialized countries, one of the main environmental problems is due to the soil contamination by heavy metals. In fact, heavy metals are nonbiodegradable and strongly toxic affecting the ecosystem and the proprieties of soil. In the paper by Tang et al., the authors propose a method reinforcing the contaminated soils with fiber and cement, and through laboratory tests, they evaluate the strength of heavy metal contaminated reinforced soil. The test results show that the mechanical properties of reinforced soil change with increasing the fiber content until an optimum content at which the maximum reinforced soil corresponds. Thus, the authors verify that the addition of fibers can effectively prevent the damage caused by freezethaw cycles to the soils.

\section{Conflicts of Interest}

The guest editors declare that there are no conflicts of interest regarding the publication of this special issue.

\section{Acknowledgments}

The guest editors wish to thank the editors for welcoming the idea of this special issue.

Donatella Termini

Hazi M. Azamathulla

Jose R. B. Cantalice 


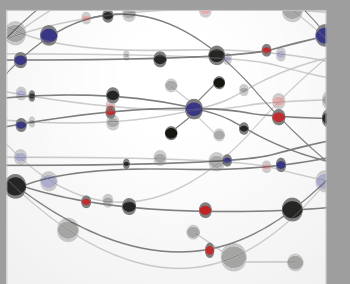

The Scientific World Journal
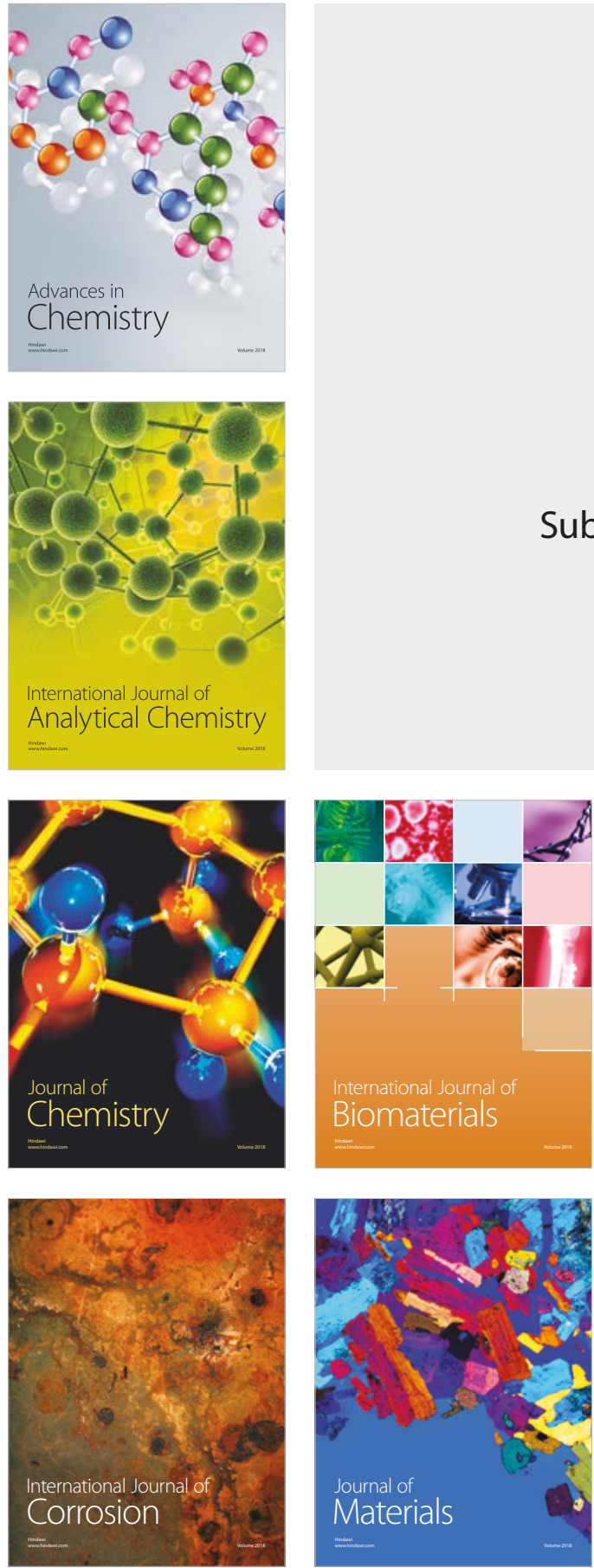

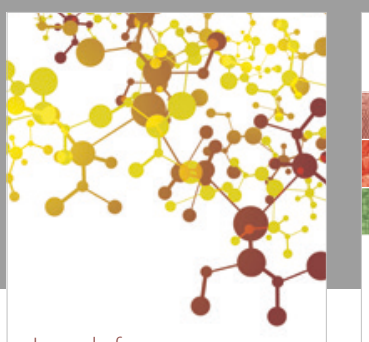

Journal of

Applied Chemistry
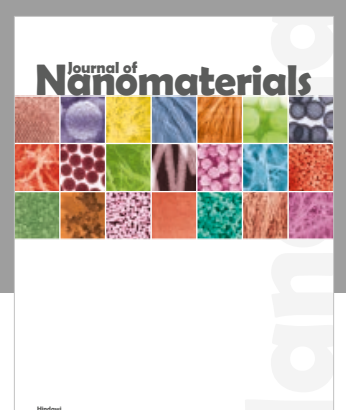

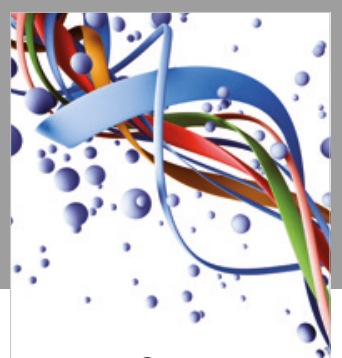

Scientifica

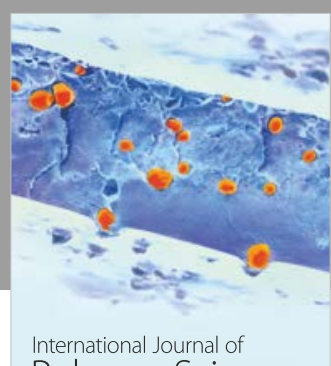

Polymer Science

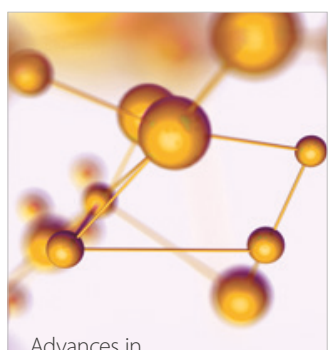

Physical Chemistry
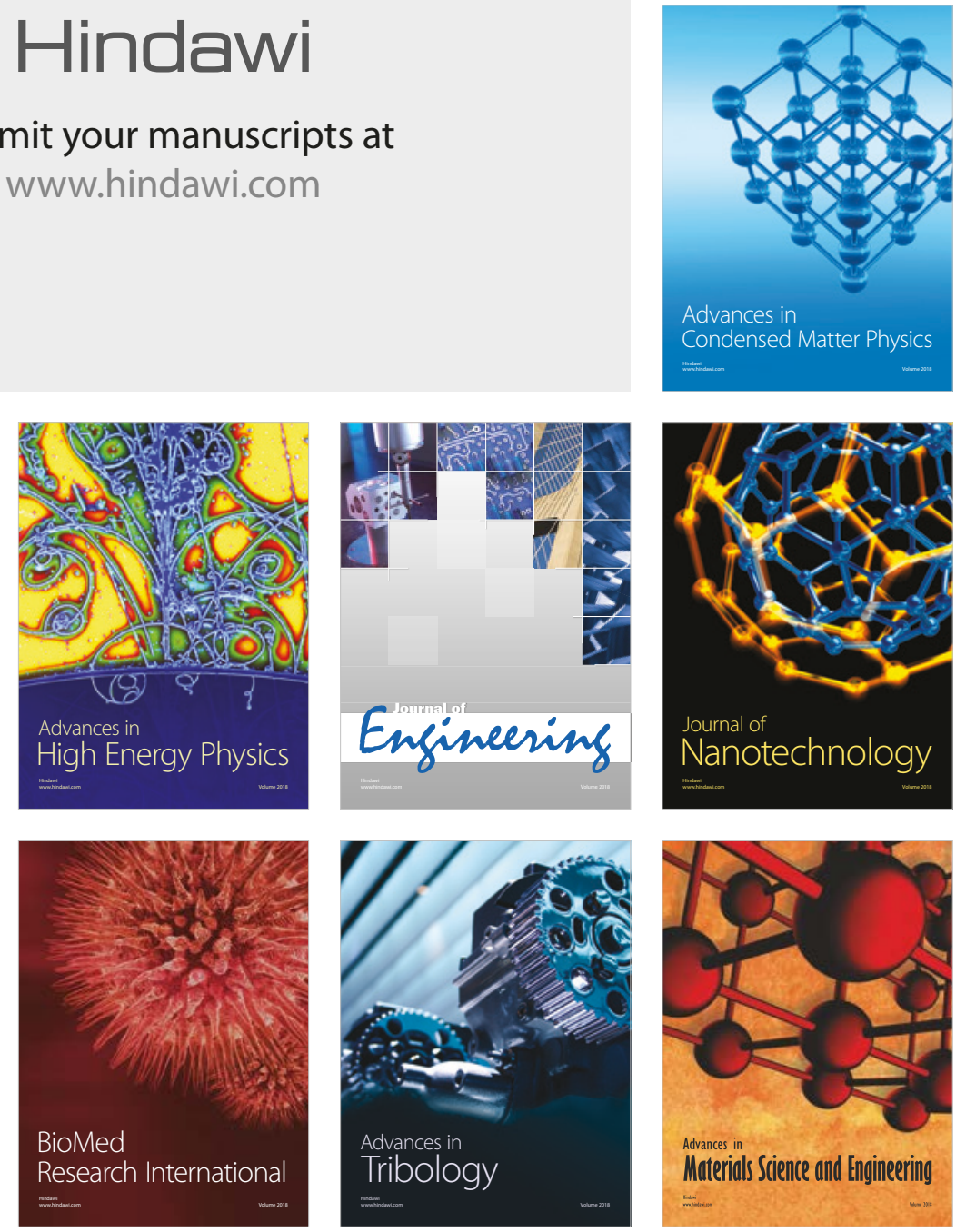